Revista Mexicana de Astronomía y Astrofísica, 55, 141-149 (2019)

DOI: https://doi.org/10.22201/ia.01851101p.2019.55.02.02

\title{
INTERSTELLAR ABSORPTION TOWARDS THE NOVAE V339 DEL AND V5668 SGR
}

\author{
D. Jack ${ }^{1,2}$ and K.-P. Schröder ${ }^{1}$ \\ Received December 27 2018; accepted March 272019
}

\begin{abstract}
We present a search and study of interstellar (IS) absorption features in the observed spectra of two Novae V339 Del and V5668 Sgr. We obtained high resolution spectra $(R \approx 20,000)$ in the wavelength range between 3800 and $8800 \AA$ of both novae with the TIGRE telescope. Common IS features of $\mathrm{Na}$ I and $\mathrm{Ca}$ II were identified in both novae, and the Ca II H and K features of Nova V339 Del show substructures, while a blueshifted absorption feature was found in the spectra of Nova V5668 Sgr. Absorption of K I was identified only in the spectra of Nova V5668 Sgr. The DIBs 6196, 6203, 6379, 6614 and 7562 were found in both novae, and in the spectra of Nova V5668 Sgr we could also identify the DIBs 5780, 5797 and 6660. We present the equivalent widths of all features and determined the column densities of Ca II, K I and hydrogen in the direction of Nova V5668 Sgr $\left(\log N_{\text {Ca II }}=12.50, \log N_{\mathrm{KI}}=11.55, \log N_{\mathrm{H}}=18.5\right)$.
\end{abstract}

\section{RESUMEN}

Presentamos una búsqueda y un estudio de características de absorción interestelar (IS) en los espectros de las novas V339 Del y V5668 Sgr. Obtuvimos espectros de alta resolución $(R \approx 20,000)$ en el intervalo de longitud de onda de 3800 a $8800 \AA$ con el telescopio TIGRE. Identificamos las características IS comunes de Na I y Ca II; las características de Ca II H y K muestran subestructuras en la Nova V339 Del, mientras que encontramos una segunda característica de absorción en los espectros de la Nova V5668 Sgr. Se identificó absorción de K I sólo en los espectros de la Nova V5668 Sgr. Las DIBs 6196, 6203, 6379, 6614 y 7562 se encontraron en ambas novas, y en los espectros de la Nova V5668 Sgr pudimos también identificar las DIBs 5780, 5797 y 6660. Presentamos los anchos equivalentes de todas las características y determinamos las densidades de columna de Ca II, K I e hidrógeno en la dirección de la nova V5668 Sgr $\left(\log N_{\text {Ca II }}=12.50, \log N_{\mathrm{KI}}=11.55\right.$, $\left.\log N_{\mathrm{H}}=18.5\right)$.

Key Words: ISM: lines and bands — novae, cataclysmic variables — techniques: spectroscopic

\section{INTRODUCTION}

Most of the interstellar and intergalactic gas present in our Universe is not directly visible. However, a suitable way of studying the interstellar medium (ISM) is to analyze the absorption features found in the spectra of bright astrophysical objects in the background. Spectra of bright stars like O-type stars or evolved red giant stars may contain interstellar absorption lines. Typically, absorption features

\footnotetext{
${ }^{1}$ Departamento de Astronomía, Universidad de Guanajuato, Guanajuato, México.

${ }^{2}$ Hamburger Sternwarte, Universität Hamburg, Hamburg, Germany.
}

of the lines of atomic species like Na I, CaII, K I and Mg II are found in observed spectra. To study these features in detail, e.g. resolving possible substructures, mid to high resolution spectroscopy of the background object is necessary.

With recent modern spectroscopy several unidentified features of interstellar absorption lines in the spectra of luminous objects have been found. These so called diffuse interstellar bands (DIBs) have been detected in high resolution spectra of massive stars (Herbig 1995; Sonnentrucker et al. 2018) and can be found in the spectra of more distant objects like 
those in the Small and Large Magellanic Clouds (Vladilo et al. 1987; Ehrenfreund et al. 2002; Cox et al. 2006, 2007; Welty et al. 2006). In a recent study of DIBs a galactic nucleus has been used as the background light source (Ritchey \& Wallerstein 2015). Further very useful and distant background light sources are extragalactic supernovae (SN) (D'Odorico et al. 1989; Sollerman et al. 2005; Cox \& Patat 2008, 2014), which offer the opportunity to study also the ISM of the respective SN host galaxy. The first study of the ISM with SN spectra was performed with the bright type II SN 1987A (de Boer et al. 1987), which exploded in the Large Magellanic Cloud. The latest very bright supernova SN 2014J offered also an excellent opportunity to study the interstellar absorption in our Galaxy and in the host galaxy M82 with high resolution spectra (Welty et al. 2014; Graham et al. 2015; Ritchey et al. 2015; Jack et al. 2015).

In our Galaxy, the outbreaks of cataclysmic variables are suitable objects for studying the interstellar material (Mauche et al. 1988; Ritchey et al. 2013). In general, events of classical novae present the opportunity to study the ISM using the absorption features in the respective nova spectra, since they are bright sources, and IS absorption features should also be visible in high resolution nova spectra. Recently, the IS absorption features in several nova spectra have been studied in the X-ray spectral range (Gatuzz et al. 2018). However, we are not aware of any publication that reports a study of the ISM using high resolution optical spectra of classical novae, motivating us to investigate our high resolution spectra of two recently observed classical novae.

In this work, we present a search and study of the absorption features of the ISM in the high resolution spectra of the two classical novae V339 Del and V5668 Sgr obtained with the TIGRE telescope. In $\S 2$, we present details about the observations that we obtained from both novae. In the following $\S 3$, we present the identification and analysis of the ISM features found in the spectra of both novae including atomic lines and DIBs. We are closing with a summary and our conclusions in the final $\S 4$.

\section{OBSERVATIONS}

\subsection{The TIGRE Telescope}

All the nova spectra used in this work have been obtained with the $1.2 \mathrm{~m}$ robotic telescope el TIGRE (Telescopio Internacional de Guanajuato Robótico Espectroscópico) situated at the La Luz Observatory in the state of Guanajuato, Central Mexico.
The design of the telescope with its automatic observation program and reduction software is ideal for obtaining time series of spectra, especially of novae and supernovae. The telescope is equipped with the HEROS (Heidelberg Extended Range Optical Spectrograph) échelle spectrograph which has a resolution of $R \approx 20,000$ and covers the optical wavelength range from about 3800 to $8800 \AA$ divided into two channels. The blue channel covers the range in wavelength from about 3800 to $5700 \AA$, while the range of the red channel goes from about 5800 to $8800 \AA$, thus leaving a small gap of roughly $100 \AA$ between the two channels. For a more detailed technical description of the hardware and software of the telescope please consult Schmitt et al. (2014). The automated wavelength calibration with a ThAr hollow cathode lamp used for our observations has a standard deviation of about $130 \mathrm{~m} \mathrm{~s}^{-1}$.

\subsection{Nova V339 Del}

Nova V339 Del (Nova Delphini 2013, PNV J20233073+2046041) was discovered on August 14 in 2013 (Nakano et al. 2013) and reached its maximum on August 16 with an apparent brightness of $V=4.46 \mathrm{mag}$ (Munari et al. 2013). The TIGRE telescope had been just recently installed at its new site, and we were able to obtain a time series of 18 spectra during the different spectral phases of that nova. Please consult De Gennaro Aquino et al. (2015) for all the details about the observations and an analysis of the spectra of Nova V339 Del obtained with the TIGRE telescope.

For our study of the ISM absorption features in this work, we added the first two spectra observed on August 17 and 19 in 2013 in order to obtain one spectrum with a higher signal-to-noise ratio. We selected only the first two spectra since the following spectra already showed stronger changes in the nova features and the continuum started to fade as well. The two added spectra were taken around maximum light during the absorption phase of Nova V339 Del.

\subsection{Nova V5668 Sgr}

On March 15 in 2015 a nova explosion was discovered in the constellation of Sagittarius (Williams et al. 2015). The Nova V5668 Sgr (Nova Sagittarii 2015 No. 2, PNV J18365700-2855420) was also a very bright event and has been studied intensively covering all parts of the electromagnetic spectrum. Using the TIGRE telescope, we obtained a long time series of optical high resolution spectra of this nova during our observation campaign of about two years 

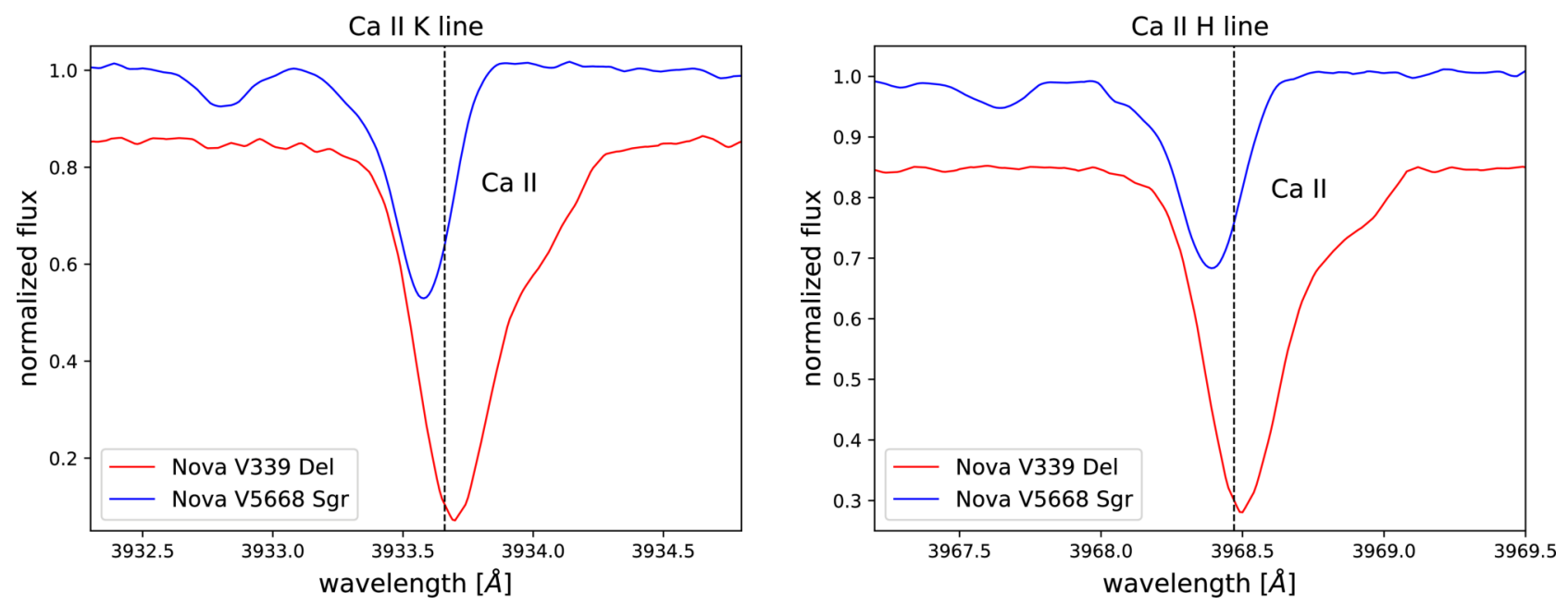

Fig. 1. Features of interstellar absorption of the Ca II H and K lines in the spectra of Novae V339 Del and V5668 Sgr. The spectra in Nova V339 Del seem to have a substructure in the red part of the main feature. The spectra of Nova V5668 Sgr show second weaker blueshifted absorption features in both lines. The vertical lines mark the rest wavelengths of the $\mathrm{H}$ and $\mathrm{K}$ lines. The color figure can be viewed online.

since this nova was of type DQ Her having a long lasting variation phase. Details about the observations and a presentation of the spectra can be found in Jack et al. (2017). The spectra of Nova V5668 Sgr are calibrated for relative fluxes.

To study the ISM absorption in the direction of Nova V5668 Sgr we added the first three spectra observed on March 19, 20 and 21 in 2015 to obtain one spectrum with a higher signal-to-noise ratio. These three spectra, observed during the absorption phase, were still similar and still showed a strong continuum flux. Adding more spectra would surely increase the signal-to-noise ratio. However, since the spectrum of a nova changes within days (or even hours), adding too many spectra may cause the appearance of false features, and complicates the continuum determination.

\section{ABSORPTION FEATURES OF THE ISM IN THE SPECTRA OF THE NOVAE V339 DEL AND V5668 SGR}

Having high resolution spectra of both novae, the goal is to identify in these spectra all absorption features of the interstellar medium. As commented in the previous section, we added several spectra to obtain one spectrum with a higher signal-to-noise ratio. All used spectra were obtained during the absorption phase so that enough continuum flux in the nova spectrum is present to be able to identify the absorption features of the interstellar gas, which can be due to absorption either by atomic lines or by diffuse interstellar bands. Nova features are very broad, having widths of up to a few $10 \AA$, which makes it easy to distinguish them from the narrow features of IS absorption that have widths of the order of only a few $0.1 \AA$.

\subsection{Interstellar Absorption of Atomic Lines}

In the first part of our analysis, we searched for the most common features of interstellar absorption by atomic lines in the spectra of both Novae V339 Del and V5668 Sgr. While the absorption features of the $\mathrm{Ca}$ II $\mathrm{H}$ and $\mathrm{K}$ lines as well as the two Na I D lines are clearly present in both novae, we could identify features of two K I lines only in Nova V5668 Sgr. However, in the spectra of Nova V339 Del they might be blended with telluric absorption lines.

Figure 1 presents a graph of the features of both the $\mathrm{Ca} I \mathrm{II}$ and $\mathrm{K}$ lines in the observed normalized spectra of both novae. The main feature in Nova V339 Del is slightly redshifted, while the main feature in Nova V5668 Sgr is found to be slightly blueshifted. The features in the spectra of Nova V339 Del seem to have a substructure meaning that they are blended with a second feature that is shifted slightly to the right of the main feature, thus, causing a slight bump around 3934.1 and 3969.0 A respectively. Both the Ca II H and K lines in Nova V5668 Sgr have a second smaller blueshifted feature at around 3932.8 and $3967.7 \AA$.

Another usually strong ISM absorption feature originates from the $\mathrm{NaI} \mathrm{D}$ lines. Figure 2 shows a graph of the spectra of Novae V339 Del and 
TABLE 1

EQUIVALENT WIDTHS AND VELOCITIES OF THE ISM*

\begin{tabular}{|c|c|c|c|c|c|}
\hline \multirow[b]{2}{*}{ Feature } & \multirow[b]{2}{*}{ Wavelength $[\AA]$} & \multicolumn{2}{|c|}{ Velocity $[\mathrm{km} / \mathrm{s}]$} & \multicolumn{2}{|c|}{ Equivalent width $[\mathrm{m} \AA]$} \\
\hline & & V339 Del & V5668 Sgr & V339 Del & V5668 Sgr \\
\hline \multirow[t]{2}{*}{ Ca II } & 3933.66 & $3.0 \pm 0.4$ & $-6.1 \pm 0.4$ & $345.3 \pm 3.4$ & $141.9 \pm 1.3$ \\
\hline & & & $-65.5 \pm 1.5$ & & $22.1 \pm 2.6$ \\
\hline \multirow[t]{2}{*}{ Ca II } & 3968.47 & $2.3 \pm 0.4$ & $-6.0 \pm 0.4$ & $221.0 \pm 1.8$ & $93.2 \pm 1.1$ \\
\hline & & & $-62.7 \pm 1.5$ & & $9.5 \pm 1.9$ \\
\hline $\mathrm{Na} \mathrm{I}$ & 5889.95 & $2.5 \pm 0.4$ & $-5.1 \pm 0.4$ & $394.9 \pm 5.3$ & $289.1 \pm 3.3$ \\
\hline $\mathrm{Na} I$ & 5895.92 & $3.1 \pm 0.4$ & $-5.1 \pm 0.4$ & $306.4 \pm 8.4$ & $247.8 \pm 1.3$ \\
\hline K I & 7664.91 & & $-5.5 \pm 0.4$ & & $46.9 \pm 1.2$ \\
\hline K I & 7698.97 & & $-5.6 \pm 0.4$ & & $80.1 \pm 0.5$ \\
\hline
\end{tabular}

*Features Found in the Spectra of Novae V339 Del and V5668 Sgr.

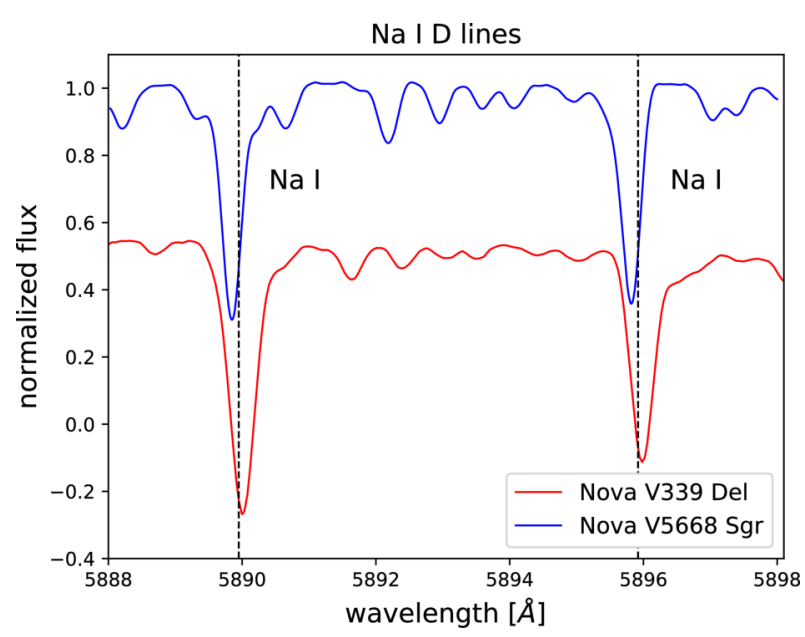

Fig. 2. Features of interstellar absorption of the Na I D lines in the spectra of Novae V339 Del and V5668 Sgr. The features are present in both novae and have about the same position when compared to the rest wavelengths (marked by vertical lines) as in the $\mathrm{Ca}$ I $\mathrm{H}$ and $\mathrm{K}$ lines. The color figure can be viewed online.

V5668 Sgr around the NaI D lines. Like for the Ca I H and K lines, the features of Nova V339 Del are slightly redshifted. However, there is no clear indication of a substructure in this feature. The features of Nova V5668 Sgr are slightly blueshifted, but we could not identify a second feature, because the region around the $\mathrm{Na}$ I D lines is contaminated with several telluric lines.

Figure 3 shows the spectrum of Nova V5668 Sgr where two features of interstellar absorption of K I lines could be identified. The features closer to the rest wavelengths are the absorption features of the ISM, which like the other IS absorption features in
Nova V5668 Sgr are slightly blueshifted. The other two features in each plot are telluric lines which belong to the A band of $\mathrm{O}_{2}$ in the Earth's atmosphere. In the spectra of Nova V339 Del the positions of both K I lines unfortunately coincide with the telluric lines, and we could therefore not confirm the presence of interstellar K I absorption in that nova.

We measured the equivalent widths of all the identified features in the spectra of Novae V339 Del and V5668 Sgr. By measuring the position of the minimum of the features, we could determine the relative velocities of the ISM features in both novae. The position of the minimum was determined by fitting a Gauss profile to the features. Table 1 presents the results of these measurements. All the features of ISM absorption in Nova V339 Del have a similar velocity between 2 and $3 \mathrm{~km} \mathrm{~s}^{-1}$ meaning that the features of all lines correspond to the same ISM cloud. All the main features of the ISM in Nova V5668 Sgr also show similar velocities between -6 and $-5 \mathrm{~km} \mathrm{~s}^{-1}$ corresponding to the same IS gas cloud. Both the Ca II $\mathrm{H}$ and $\mathrm{K}$ lines have a second absorption feature with velocities of -65.5 and $-62.7 \mathrm{~km} \mathrm{~s}^{-1}$ respectively, so that a second IS gas cloud could lie in the direction towards Nova V5668 Sgr. However, we could not observe this feature in the Na I D lines, maybe also because there are several telluric absorption features in this wavelength range.

The IS absorption features in Nova V339 Del show in general greater equivalent widths than the ones in Nova V5668 Sgr. As is usually found for interstellar absorption, the features of $\mathrm{Na} I$ are the strongest in the spectra of both Novae. As can also 
KI line at $7664.91 \AA$

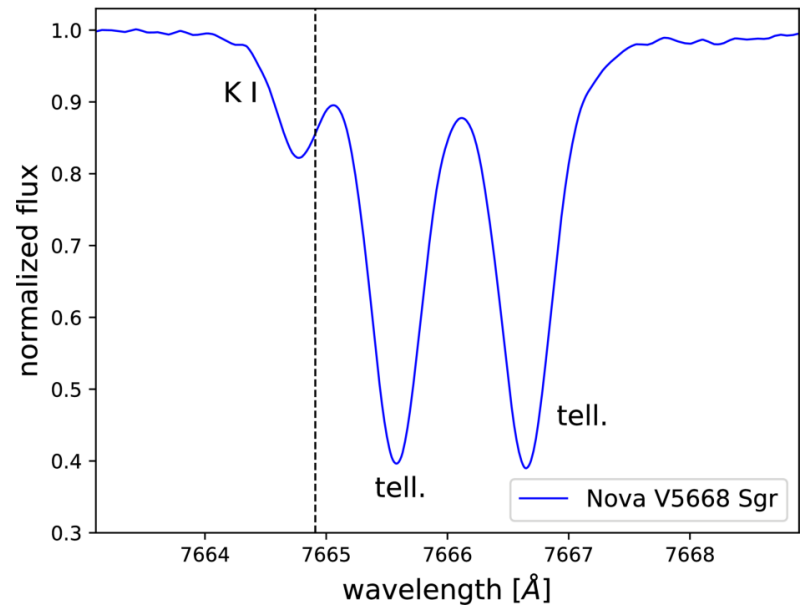

K I line at $7698.97 \AA$

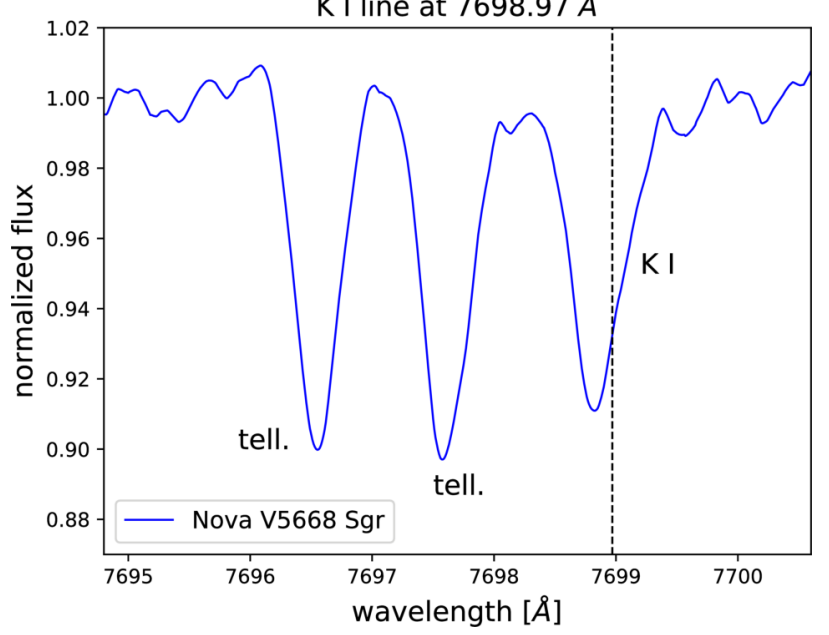

Fig. 3. Features of interstellar absorption of two K I lines in the spectra of Nova V5668 Sgr. The rest wavelengths are marked by vertical lines. The two stronger features in each plot come from telluric line absorption. The color figure can be viewed online.

be seen in Figures 1 and 2 the IS absorption features of $\mathrm{Ca}$ II and $\mathrm{Na}$ I are saturated in the spectra of Nova V339 Del.

Using the analysis of pure absorption lines, formerly applied by us to special problems in the field of stellar atmospheres (Schroeder et al. 1994), we fitted the IS absorption features of Ca II and K I of Nova V5668 Sgr assuming optically thin lines. The Ca II H and K lines of Nova V5668 Sgr are ideal to determine both the column density $N_{\mathrm{Ca} \text { II }}$ and the turbulence velocity $v_{\text {tur }}$ as they are intermediateoptically thick. By contrast, in Nova V339 Del this doublet is already very saturated and unfavorable to derive either parameter. The same is true, in both Novae, for the very strong Na I D doublet. In our analysis, we determined a turbulence velocity for the ISM of $v_{\text {tur }}=4.5 \mathrm{~km} \mathrm{~s}^{-1}$. For the $\log (f g)$-values of the $\mathrm{Ca}$ II $\mathrm{K}$ and $\mathrm{H}$ lines we used 0.69 and 0.34 respectively (Wiese et al. 1969). The $\log (f g)$-values for both K I lines are 0.59 and 0.29 respectively (Nandy et al. 2012).

With the analysis of the depth and profile of the Ca II features assuming small saturation and solving for the best fit of the two parameters we obtained a column density for the ground level of Ca II of $\log N_{\text {Ca II }}=12.50 \pm 0.05$ and a turbulence velocity of the ISM of $v_{\text {turb }}=4.5 \mathrm{~km} \mathrm{~s}^{-1}$ reproducing well the line profiles and equivalent widths. The obtained ISM turbulence velocity matches well the values obtained by Wilson et al. (2011). Analyzing the KI features with the same method we found a column density of $\log N_{\mathrm{KI}}=11.55 \pm 0.05$ with the same determined turbulence velocity. Assuming that all the potassium is found in the K I state (low ionization), we obtained for the column density of calcium a value of $\log N_{\mathrm{Ca}}=12.8$ assuming solar abundances (Reddy et al. 2003). Comparing this value with the measured value for the column density of Ca II of $\log N_{\mathrm{Ca} \text { II }}=12.5$, we can estimate that the ionization ratio of $\mathrm{Ca}$ II to $\mathrm{Ca} \mathrm{I}$ is about 1:1. Assuming again solar abundances, the value of the column density of $\mathrm{Ca}$ of $\log N_{\mathrm{Ca}}=12.8$ corresponds to a column density of hydrogen of about $\log N_{\mathrm{H}}=18.5$ which results in a very small extinction of about $O(0.01)$.

\subsection{Diffuse Interstellar Bands}

Apart from the common absorption features of atomic lines, spectra may contain much weaker absorption features of still unknown origin, of so called diffuse interstellar bands (DIBs). Since the spectra of Novae V339 Del and V5668 Sgr have a good signalto-noise ratio and a high resolution we searched in the spectra of both novae for features of the commonly known DIBs. For this analysis we made use of published catalogs (Jenniskens \& Desert 1994; Galazutdinov et al. 2000; Hobbs et al. 2008, 2009) for the identification of the DIBs in our spectra.

Figure 4 shows the normalized spectrum of Nova V5668 Sgr in the wavelength range of the DIBs 5780 and 5797, which are strong DIBs and are commonly observed in the spectra of luminous objects. DIB 5780 is also the strongest DIB feature in the spectra of Nova V5668 Sgr. However, concerning the spectra of Nova V339 Del this wavelength region was unfor- 
TABLE 2

EQUIVALENT WIDTHS (EW) AND VELOCITIES (VEL) OF THE DIB*

\begin{tabular}{crrrrr}
\hline & \multicolumn{2}{c}{ Nova V339 Del } & & \multicolumn{2}{c}{ Nova V5668 Sgr } \\
Feature & Vel. $[\mathrm{km} / \mathrm{s}]$ & EW $[\mathrm{m} \AA]$ & & Vel. $[\mathrm{km} / \mathrm{s}]$ & EW $[\mathrm{m} \AA]$ \\
\cline { 1 - 1 } \cline { 5 - 6 } DIB 5780 & & & & $1.6 \pm 2.6$ & $180.9 \pm 2.1$ \\
DIB 5797 & & & $-1.6 \pm 3.1$ & $42.95 \pm 1.2$ \\
DIB 6196 & $2.0 \pm 1.0$ & $12.0 \pm 2.8$ & & $-5.8 \pm 1.5$ & $17.8 \pm 0.6$ \\
DIB 6203 & $0.2 \pm 1.9$ & $33.8 \pm 4.7$ & & $-6.8 \pm 2.2$ & $25.6 \pm 1.3$ \\
DIB 6379 & $-0.8 \pm 1.0$ & $8.2 \pm 2.5$ & & $-4.7 \pm 1.4$ & $31.0 \pm 2.6$ \\
DIB 6614 & $2.9 \pm 2.2$ & $30.6 \pm 4.3$ & & $-10.9 \pm 5.4$ & $70.2 \pm 4.6$ \\
DIB 6660 & & & $-2.3 \pm 3.6$ & $12.9 \pm 1.4$ \\
DIB 7562 & $-19.4 \pm 5.4$ & $21.1 \pm 4.1$ & & $-9.5 \pm 4.3$ & $30.3 \pm 2.3$ \\
\hline
\end{tabular}

*Features found in Novae V339 Del and V5668 Sgr.

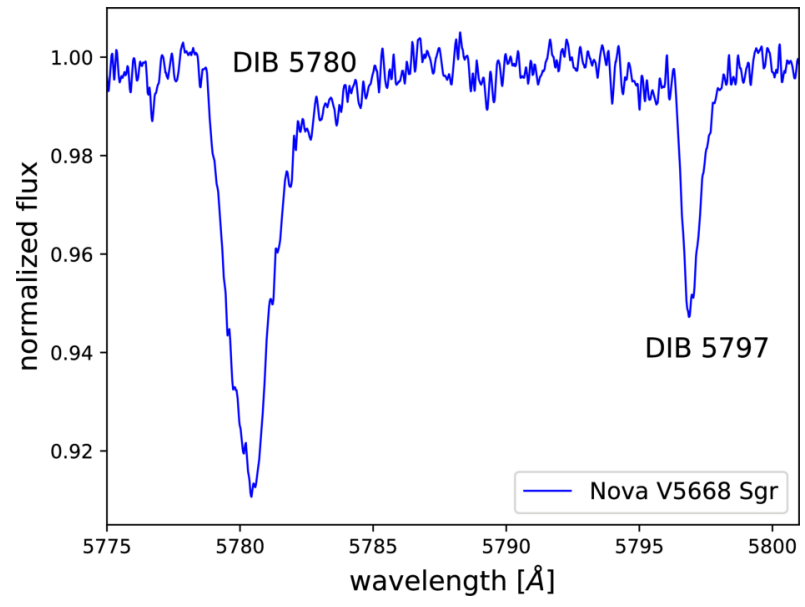

Fig. 4. Normalized spectrum of Nova V5668 Sgr showing the absorption features of the two strong DIBs 5780 and 5797. The color figure can be viewed online.

tunately in the gap between the two channels of the HEROS spectrograph. Thus, we could not look for features of the DIBs 5780 and 5797 in the spectra of Nova V339 Del.

We found features of the DIBs 6196, 6203, 6379 and 7562 in the spectra of both Novae. In Figure 5, we show in subplot (a) the normalized spectra in the wavelength region around the DIBs 6196 and 6203. The DIBs of Nova V5668 Sgr are slightly shifted to the blue when compared to the features of Nova V339 Del. This is in good agreement with the observation of the atomic IS absorption lines where the features of V5668 Sgr are also slight shifted to the blue when compared to Nova V339 Del. The subplots (b) and (c) of Figure 5 show the DIBs 6379 and 6614 respec- tively. Both DIBs in the spectra of Nova V5668 Sgr are also slightly blueshifted compared to the features of Nova V339 Del. The DIB 6660 is a relatively weak DIB, and we could clearly identify it only in the spectra of Nova V5668 Sgr, as can be seen in subplot (d) of Figure 5. The weakest feature was detected for DIB 7562 and is shown in subplot (e).

We normalized all spectra of the Novae V339 Del and V5668 Sgr using a spline fit to the continuum around the DIB features. The velocities of the features were determined using the rest wavelengths published in the catalog of Galazutdinov et al. (2000), and we measured the respective equivalent widths. We present in Table 2 the results and give the values of the velocity (in $\mathrm{km} / \mathrm{s}$ ) and equivalent width (in $m \AA \AA$ ) of all identified DIB features. The strongest feature in Nova V5668 Sgr is the DIB 5780, while in Nova V339 Del the DIB 6203 is the strongest. The weakest feature in terms of the equivalent width in Nova V5668 Sgr is the DIB 6660. The velocities for Nova V5668 Sgr are widely spread from -10.9 to $1.6 \mathrm{~km} \mathrm{~s}^{-1}$, which is probably due to the unknown origin and rest wavelengths of the DIBs and the difficulty of determining the exact position of the minimum due to the low signal-to-noise ratio of the observed features. However, the velocities of the DIB features roughly coincide with the blueshifted atomic ISM absorption features. For Nova V339 Del the velocities range from -0.8 to $2.9 \mathrm{~km} \mathrm{~s}^{-1}$ with the exception of DIB 7562 where the determination of the velocity is difficult and the measurements gave a velocity of $-19.4 \mathrm{~km} \mathrm{~s}^{-1}$. These velocities also coincide roughly with the values measured for the atomic ISM features, which are also slightly redshifted. 


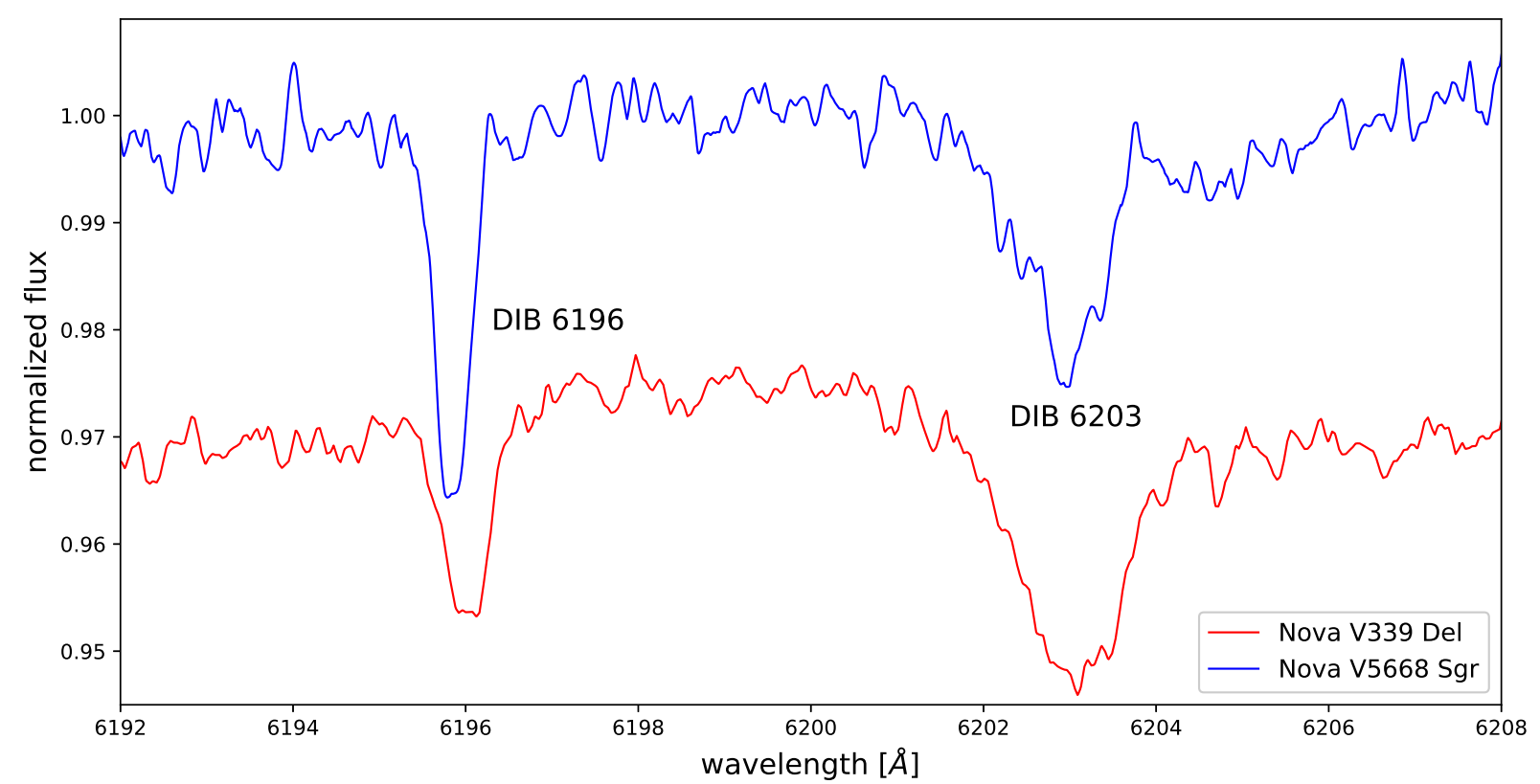

(a)

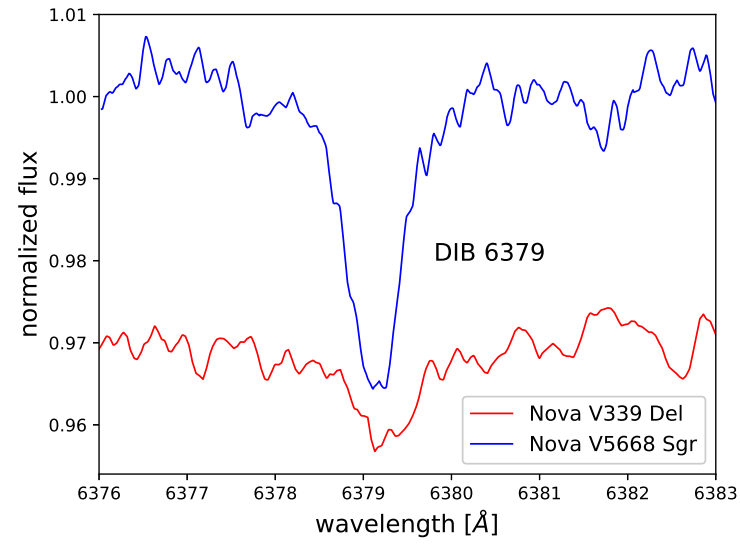

(b)

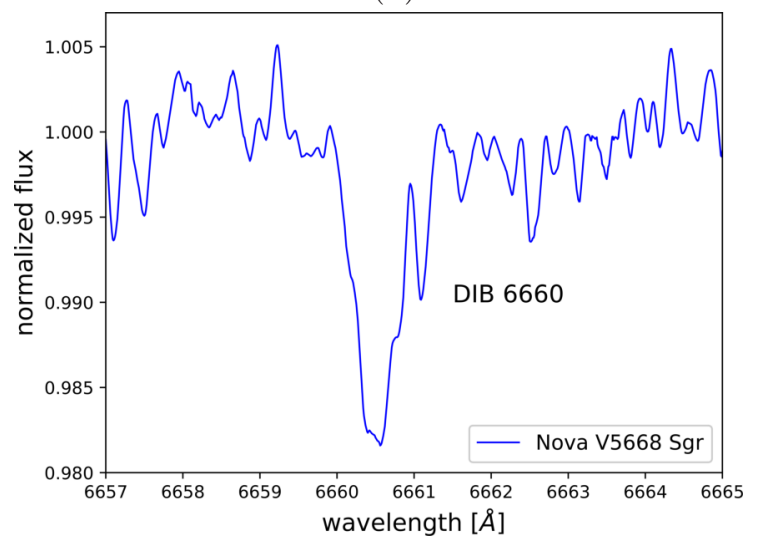

(d)

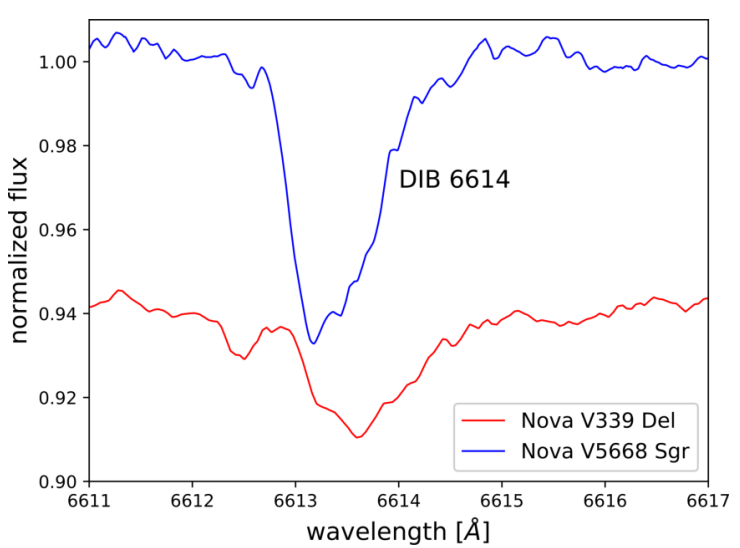

(c)

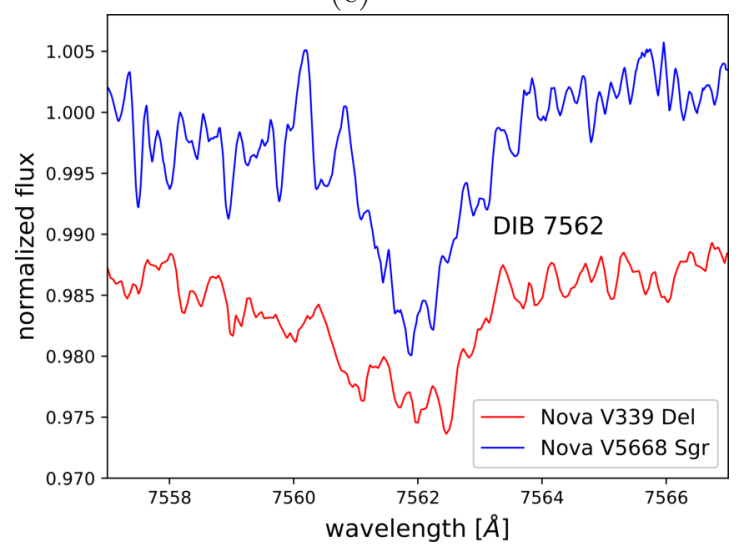

(e)

Fig. 5. Normalized spectra of Novae V339 Del and V5668 Sgr in the wavelength range of the DIBs 6196, 6203, 6379, 6614, 6660 and 7562. The spectra of Nova V339 Del are shifted for a better illustration. The color figure can be viewed online. 


\section{SUMMARY AND CONCLUSIONS}

We used high resolution spectra $(R \approx 20,000)$ of the two bright novae, V339 Del and V5668 Sgr, observed with the TIGRE telescope to search for signs of interstellar absorption by both atomic lines and diffuse interstellar bands. We were able to identify several features of interstellar absorption by atomic lines as well as DIBs and we measured the velocities and equivalent widths of these features.

We found in the spectra of both novae interstellar absorption features of the $\mathrm{Ca}$ II $\mathrm{H}$ and $\mathrm{K}$ lines. The detected features in the spectra of Nova V339 Del seem to show substructures having a second, blended, weaker and slightly redshifted feature. Apart from the main slightly blueshifted features, we found in the spectra of Nova V5668 Sgr additional weaker and blueshifted features with velocities of -65.5 and $-62.7 \mathrm{~km} \mathrm{~s}^{-1}$ respectively. Absorption features observed in the atomic lines of Na I D show velocities similar to Ca II in both novae, meaning that they originate from the same IS gas cloud. However, we could not identify substructures or secondary features. Evidence for absorption of K I lines could only be found in the spectra of Nova V5668 Sgr. Two features of the lines with rest wavelengths of 7664.91 and $7698.97 \AA$ are present. However, in Nova V339 Del these features might be hidden in the telluric lines. The main features of Nova V339 Del have velocities between 2 and $3 \mathrm{~km} \mathrm{~s}^{-1}$, while the main features of Nova V5668 Sgr are blueshifted showing velocities between -6 and $-5 \mathrm{~km} \mathrm{~s}^{-1}$. Consulting the literature we found that the ISM absorption in the emission-line star 9 Sgr has components at velocities of $\approx-62$ and $\approx-10 \mathrm{~km} \mathrm{~s}^{-1}$ (Pwa \& Pottasch 1986). The ISM absorption towards the Galactic Center in Sgr shows, among others, foreground arms with velocities of $\approx-55$ and $\approx-5 \mathrm{~km} \mathrm{~s}^{-1}$ (Oka et al. 1998). This all agrees well with our velocity determination of both observed ISM components in our spectra of Nova V5668 Sgr.

The ISM absorption features in Nova V339 Del are in general stronger with greater equivalent widths when compared to the ones in Nova V5668 Sgr. In both novae, the IS absorption of $\mathrm{NaI}$ is strongest. The atomic IS features in Nova V339 Del are all saturated. However, for Nova V5668 Sgr using the IS absorption of Ca II and $\mathrm{K} I$ we could reproduce the equivalent widths and profiles of the features with a turbulence velocity for the ISM of $v_{\text {tur }}=4.5 \mathrm{~km} \mathrm{~s}^{-1}$ and column densities for Ca II of $\log N_{\mathrm{CaII}}=12.50 \pm 0.05$ and for K I of $\log N_{\mathrm{KI}}=11.55 \pm 0.05$. Assuming solar abundances we determined the column density of hydrogen to be about $\log N_{\mathrm{H}}=18.5$, which corresponds to low extinction.

We also performed an identification of diffuse interstellar bands in the spectra of both novae. The DIBs 6196, 6203, 6379, 6614 and 7562 could be detected in both novae. The DIB 6660 shows a weak feature only in the spectra of Nova V5668 Sgr. The DIBs 5780 and 5797 could only be identified in the Nova V5668 Sgr, because in the spectra of Nova V339 Del these DIBs fall into the gap between the two channels of the HEROS spectrograph. By determining the velocities we found that the DIBs of Nova V5668 Sgr are slightly blueshifted, which is in good agreement with the measured velocities of the ISM absorption features in the atomic lines, meaning that the DIBs have probably the same origin as the atomic lines. The equivalent widths of the DIB features in the spectra of Novae V339 Del and V5668 Sgr were presented.

In this work, we demonstrated that high resolution spectroscopy of classical novae offers a further opportunity to study the interstellar medium since the absorption features of both atomic lines and diffuse interstellar bands can be found and studied in detail.

This research has been made possible in part by grants of the Universidad de Guanajuato (UG) through the DAIP project CIIC 021/2018 and also by CONACYT in several bilateral grants. D. Jack would like to thank the staff of the Hamburger Sternwarte for the hospitality during his sabbatical stay.

\section{REFERENCES}

Cox, N. L. J., Cordiner, M. A., Cami, J., et al. 2006, A\&A, 447, 991

Cox, N. L. J., Cordiner, M. A., Ehrenfreund, P., et al. 2007, A\&A, 470, 941

Cox, N. L. J. \& Patat, F. 2008, A\&A, 485, L9 2014, A\&A, 565, A61

de Boer, K. S., Richtler, T., \& Savage, B. D. 1987, ESO Workshop on the SN 1987A, (Germany: ESO) 26, 549

De Gennaro Aquino, I., Schröder, K.-P., Mittag, M., et al. 2015, A\&A, 581, A134

D'Odorico, S., di Serego Alighieri, S., Pettini, M., et al. 1989, A\&A, 215, 21

Ehrenfreund, P., Cami, J., Jiménez-Vicente, J., et al. 2002, ApJ, 576, L117

Galazutdinov, G. A., Musaev, F. A., Krełowski, J., \& Walker, G. A. H. 2000, PASP, 112, 648

Gatuzz, E., Ness, J.-U., Gorczyca, T. W., et al. 2018, MNRAS, 479, 2457 
Graham, M. L., Valenti, S., Fulton, B. J., et al. 2015, ApJ, 801, 136

Herbig, G. H. 1995, ARA\&A, 33, 19

Hobbs, L. M., York, D. G., Snow, T. P., et al. 2008, ApJ, 680,1256

Hobbs, L. M., York, D. G., Thorburn, J. A., et al. 2009, ApJ, 705, 32

Jack, D., Mittag, M., Schröder, K.-P., et al. 2015, MNRAS, 451, 4104

Jack, D., Robles Pérez, J. d. J., De Gennaro Aquino, I., et al. 2017, AN, 338, 91

Jenniskens, P. \& Desert, F.-X. 1994, A\&AS, 106, 39

Mauche, C. W., Raymond, J. C., \& Cordova, F. A. 1988, ApJ, 335, 829

Munari, U., Henden, A., Dallaporta, S., \& Cherini, G. 2013, IBVS, 6080

Nakano, S., Itagaki, K., Denisenko, D., et al. 2013, CBET, 3628

Nandy, D. K., Singh, Y., Shah, B. P., \& Sahoo, B. K. 2012, PhRvA, 86, 2517

Oka, T., Hasegawa, T., Sato, F., Tsuboi, M., \& Miyazaki, A. 1998, ApJS, 118, 455

Pwa, T. H. \& Pottasch, S. R. 1986, A\&A, 164, 116

Reddy, B. E., Tomkin, J., Lambert, D. L., \& Allende Prieto, C. 2003, MNRAS, 340, 304
Ritchey, A. M. \& Wallerstein, G. 2015, PASP, 127, 223

Ritchey, A. M., Wallerstein, G., \& McKeever, J. 2013, PASP, 125, 1429

Ritchey, A. M., Welty, D. E., Dahlstrom, J. A., \& York, D. G. 2015, ApJ, 799, 197

Schmitt, J. H. M. M., Schröder, K.-P., Rauw, G., et al. 2014, AN, 335, 787

Schroeder, K.-P., Griffin, R. E. M., \& Hunsch, M. 1994, A\&A, 288, 273

Sollerman, J., Cox, N., Mattila, S., et al. 2005, A\&A, 429,559

Sonnentrucker, P., York, B., Hobbs, L. M., et al. 2018, ApJS, 237, 40

Vladilo, G., Crivellari, L., Molaro, P., \& Beckman, J. E. 1987, A\&A, 182, L59

Welty, D. E., Federman, S. R., Gredel, R., Thorburn, J. A., \& Lambert, D. L. 2006, ApJS, 165, 138

Welty, D. E., Ritchey, A. M., Dahlstrom, J. A., \& York, D. G. 2014, ApJ, 792, 106

Wiese, W. L., Smith, M. W., \& Miles, B. M. 1969, NSRDS-NBS, 22, 268

Williams, S. C., Darnley, M. J., \& Bode, M. F. 2015, ATel, 7230, 1

Wilson, C. D., Warren, B. E., Irwin, J., et al. 2011, MNRAS, 410, 1409

D. Jack and K.-P. Schröder: Departamento de Astronomía, Universidad de Guanajuato, A.P. 144, 36000 Guanajuato, Gto., México (dennis, kps@astro.ugto.mx).

D. Jack: Hamburger Sternwarte, Universität Hamburg, Hamburg, Germany. 